\title{
Pelvic osteomyelitis in a 15-year-old girl. Case report
}

\author{
Tugce Tural Kara M.D. ${ }^{a}$, Halil Ozdemir M.D. ${ }^{a}$, Suat Fitoz M.D. ${ }^{b}$, Ergin Ciftci M.D. ${ }^{a}$ and Erdal Ince M.D. ${ }^{a}$
}

\begin{abstract}
Pelvic osteomyelitis is a rare infection. Ilium, ischium, pubis and acetabulum are commonly affected sites. Pelvic radiography, pelvic magnetic resonance and scintigraphy are usefulimaging techniques for diagnosis. However, diagnoses should be confirmed with bone biopsy from the lesion. Sometimes diagnosis is delayed because of deep localization of infection site. Here in, we report a 15-year-old girl with left groin pain who was diagnosed as pelvic osteomyelitis one month after initial symptoms. Patient was successfully treated with antibiotics for 8 weeks. In conclusion, although pelvic osteomyelitis is an unusual infection in childhood, it should be considered as differential diagnoses of pain in groin, hips, thigh, abdomen and spine. If diagnosis is suspicious, empiric treatment should be started quickly, because it will be possible to achieve satisfactory clinical results with effective treatment.

Key words: children, pain, acute pelvic osteomyelitis.
\end{abstract}

http:/ /dx.doi.org/10.5546/aap.2016.eng.e370

\section{INTRODUCTION}

Osteomyelitis is a local infection of bone which is frequently caused by seeding of microorganisms to the bone by hematogenous way. Direct inoculation by trauma or surgical procedures and local invasion from infected soft tissue are other predisposing factors. In children the incidence is estimated to be 1 in 5000 to 7700 in developed countries, and 1 in 500 to 2300 in developing countries. ${ }^{1}$ Acute hematogenous osteomyelitis is typically seen in long tubular bones of children. ${ }^{2}$ Boys are more affected than girls and majority of children with osteomyelitis are younger than 5 years. ${ }^{3}$

a. Department of Pediatric Infectious Diseases.

b. Department of Pediatric Radiology.

Ankara University Medical School, Ankara, Turkey.

E-mail Address:

Tugce Tural Kara: tugcetural@hotmail.com

Funding: None.

Conflict of interest: None.

Received: 2-29-2016

Accepted: 4-21-2016
Pelvic osteomyelitis is rare in childhood, but it is an important disease due to complications in untreated cases. Patients may admit to hospital with pain of hips, thigh, abdomen or spine and difficulty in walking. ${ }^{4}$ Diagnosis is sometimes delayed because of deep localization of the infection site. ${ }^{2}$ In addition there are no specific clinical signs and symptoms to rule out other orthopaedic and infectious diseases. ${ }^{5}$

Here in, we report a girl with pelvic osteomyelitis who was diagnosed one month after initial symptoms.

\section{CASE REPORT}

A 15-year-old girl was admitted to hospital with complaint of left groin pain without history of fever or trauma. Pain was constant during rest and movement, unresponsive to analgesics and lasted for approximately one month. Patient had no complaint at right side of hip and groin, groin pain was more intense on left side reffering to left knee. No other joints were involved and she lost $5 \mathrm{~kg}$ of weight in last one month. Patient was treated with analgesics and muscle relaxants during admission to other centers and unresponsive to those treatments.

On physical examination, her vital signs were as follows; body temperature: $38.0^{\circ} \mathrm{C}$, heart rate: 108 beats/min, blood pressure: $100 / 70 \mathrm{mmHg}$. She could not walk without support and could not stand on her left foot. She had limited passive range of motion (flexion, abduction and external rotation) of the left hip. Patient's laboratory test results were as follows; hemoglobin: $11.6 \mathrm{~g} / \mathrm{dl}$, leukocyte count: $11000 / \mathrm{mm}^{3}$ (neutrophils $76 \%$, lymphocytes $18 \%$ and monocytes $6 \%$ ), platelet count: $571,000 / \mathrm{mm}^{3}$, erythrocyte sedimentation rate (ESR): $130 \mathrm{~mm} / \mathrm{h}$ and C-reactive protein (CRP): $19.2 \mathrm{mg} / \mathrm{dl}$. There were no pathological findings on pelvic radiography (Figure 1). Bone marrow edema of ischium and muscles were detected with pelvic magnetic resonance imaging (MRI) (Figure 2). We considered osteomyelitis and malignant infiltrative process with these findings. Bone marrow aspiration was performed and it was normal. Pathological uptake was found in symphysis pubis, lower part of the left sacroiliac joint, left ischium and left acetabulum with bone scintigraphy (Figure 3). Patient was diagnosed 
as osteomyelitis and treated with intravenous ceftriaxone. On follow-up, she was able to walk more comfortable and pain reduced on left hip movements.

On the $7^{\text {th }}$ day of antibiotic treatment patient felt mild pain on her left hip. Fever revealed above $38^{\circ} \mathrm{C}$ discontinuously without determined focus. Acute phase reactants persisted high (ESH: $130 \mathrm{~mm} / \mathrm{h}$ and CRP: $11.6 \mathrm{mg} / \mathrm{dl}$ ). For differential diagnosis of fever without focus, tests for tuberculosis and connective tissue diseases (purified protein derivative: $2 \mathrm{~mm}$, antistreptolysin $\mathrm{O}$ titer: $365 \mathrm{U} / \mathrm{L}$, rheumatoid factor: $8.44 \mathrm{mg} / \mathrm{dl}$, antinuclear antibodies: negative, Brucella agglutination: negative) were evaluated and found in normal range. There was a slight expansion of the lesion site on control pelvic MRI. To rule out malignancy or other infiltrative bone disease, biopsy and curetage of the lesion was performed. Biopsy material culture was unremarkable. Tuberculosis polymerase chain reaction, acid resistant bacillus and cultuvation of biopsy material were negative. Consequently diagnosis of osteomyelitis was confirmed with pathological examination of biopsy material. Intravenous ceftriaxone treatment was prolonged for 4 weeks. Oral ciprofloxacin and amoxicillinclavulanate treatment were given for 4 weeks after discharge. At the end of 10 months of follow-up, patient completely recovered without sequelae.

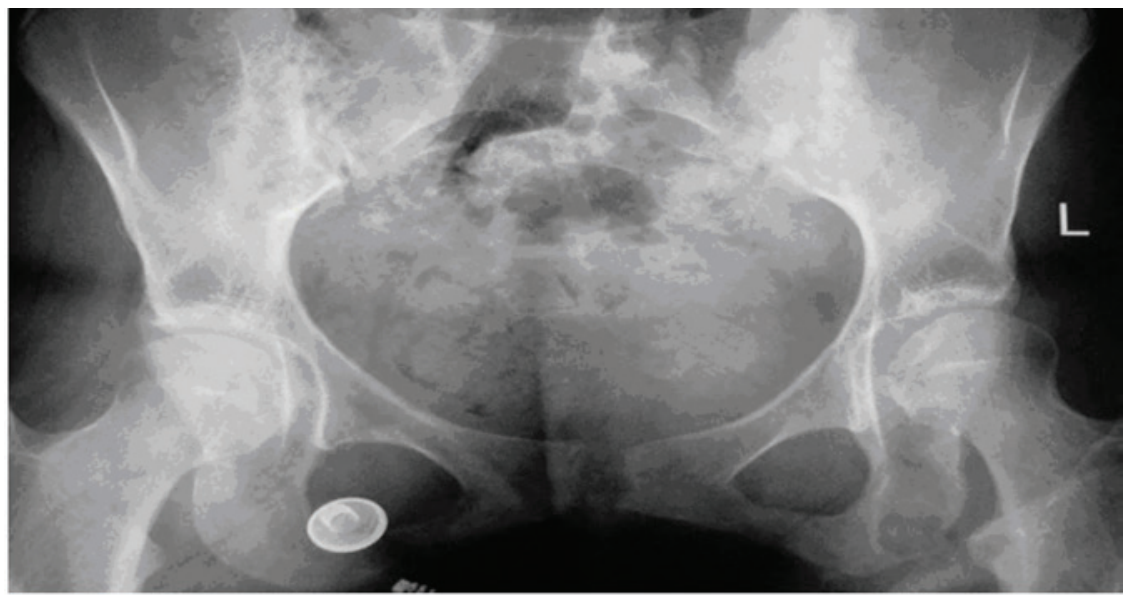

FIGURE 2. Axial T2-weighted MR image shows hyperintense signal changes on ischial bone (arrow) and muscles (curved arrow)

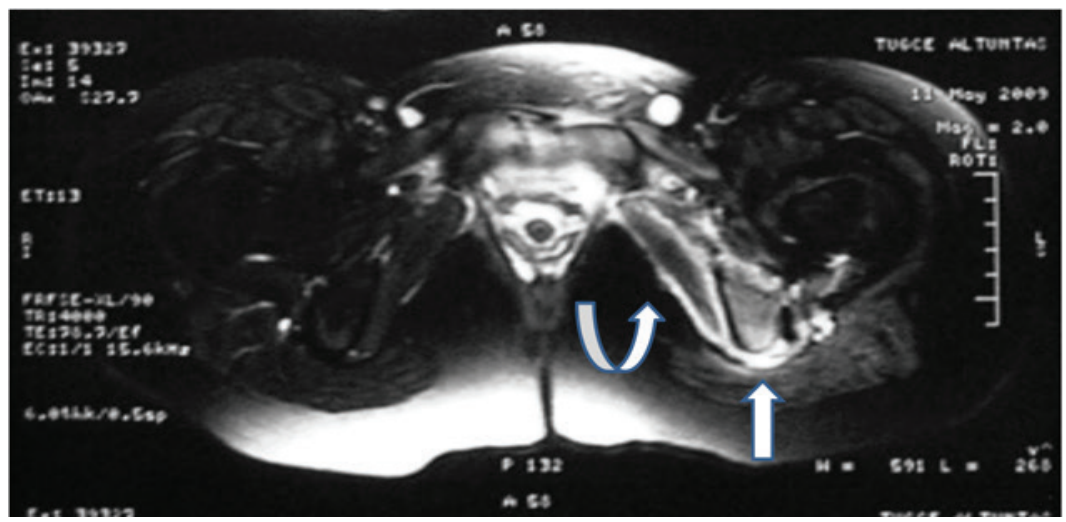




\section{DISCUSSION}

Hematogenous osteomyelitis of the pelvic bone is an unusual pediatric infection $(6.3-20 \%){ }^{2}$ Previous studies suggested that time between the onset of symptoms and admission to hospital is so long and diagnosis is often delayed due to deep localization of the infection site. ${ }^{2,6}$ Age spectrum of children with pelvic osteomyelitis is older than children with acute hematogenous osteomyelitis. ${ }^{7}$ Although pelvic osteomyelitis is more commonly seen in boys, male/female ratio is lower than acute hematogenous osteomyelitis $(1.5 / 1) .{ }^{8}$ Some factors such as pelvic procedures, urinary tract infections, trauma, Crohn disease may predispose development of osteomyelitis. Most commonly affected bone is ilium (38\%) because of its rich vascular supply ischium (19\%), pubis (14\%) and acetabulum (12\%) follows. ${ }^{4}$ In this case, patient was diagnosed as osteomyelitis one month after the beginning of symptoms. By comparison with findings in the literature we found features like age, gender, and bone involvement site of this case is rare.

History of trauma is reported as $17 \%$ in pelvic osteomyelitis, but this rate is about $40 \%$ in osteomyelitis of long bones. This supports the fact that long bones are more prone to trauma exposure compared with pelvic bone. ${ }^{8}$ Patients with pelvic osteomyelitis may present with various clinical signs. This leads to misdiagnosis and delayed diagnosis. Most common presenting complaints are fever and pain. Weight loss, anorexia, limp or decreased weight bearing may be seen in children. ${ }^{2}$ Pain usually occurs on hips, thighs or abdomen.

Blood or bone aspirate material cultures may elucidate $50 \%$ of causative microorganism. Staphylococcus aureus is the most common causative microorganism $(90 \%)$, which is similar to acute hematogenous osteomyelitis. ${ }^{4}$ Haemophilus influenzae, Salmonella spp., Group A Streptococcus, Enterobacter cloacae and Pseudomonas aeruginosa may be responsible for pelvic osteomyelitis rarely. ${ }^{9}$ Effective empirical treatment should be started against $S$. aureus immediately. Intravenous cephalosporins and clindamycin are the most commonly used treatment agents. If the responsible microorganisms are isolated, treatment should be rearranged against them. Detection of bone involvement with pelvic radiography is difficult in early stages of disease. Sensitivity of bone scintigraphy is higher. Pelvic MRI is the best diagnostic imaging modality which is $82-100 \%$ sensivitive and $75-96 \%$ specific for diagnosis. ${ }^{10}$ Prognosis of pelvic osteomyelitis is so good. Recovery rate without sequelae is over $95 \%$ but this rate decreases with delayed diagnosis and treatment failure. Recurrent osteomyelitis and deformities may develop unfortunately.

Local symptoms of inflammation, positive blood or bone aspiration culture, presence of subperiostal purulent material and typical radiological or scintigraphic changes support the diagnosis of acute hematogenous osteomyelitis. ${ }^{10}$ Our findings were sufficient for diagnosis in this case.

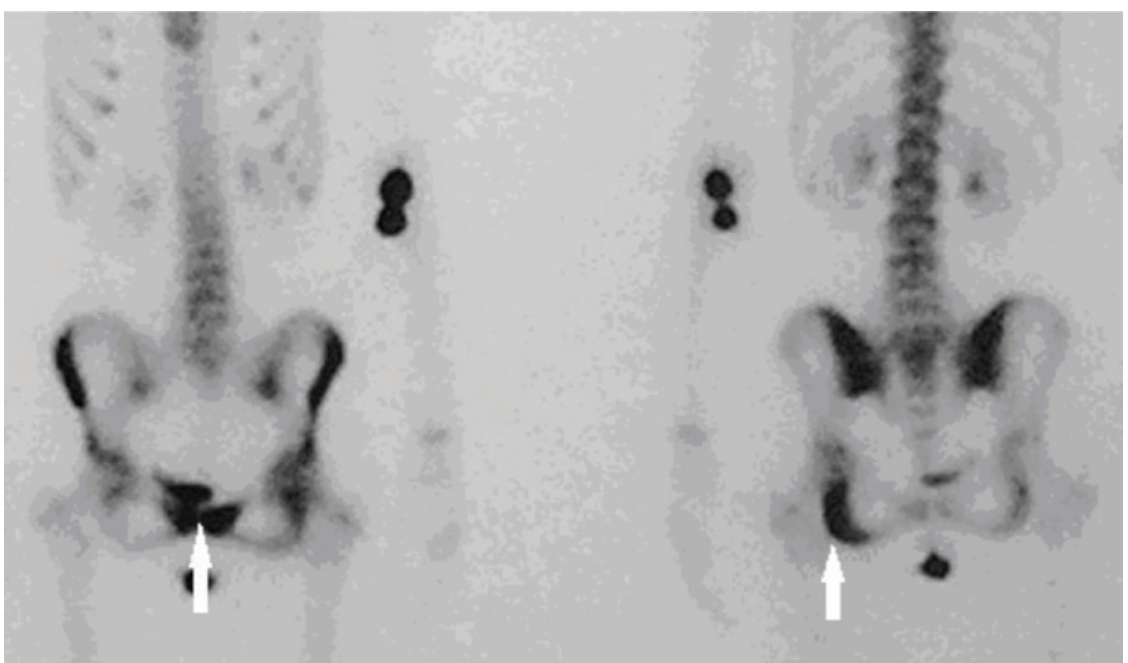


In conclusion, pelvic osteomyelitis is rare in children but it should be kept in mind for differential diagnosis of patients with hip and groin pain. If it's suspected, empiric treatment should be started immediately. Frequency of sequelae may decline to very low rates with efficient treatment.

\section{REFERENCES}

1. Dartnell J, Ramachandran M, Katchburian M. Haematogenous acute and subacute paediatric osteomyelitis: a systematic review of the literature. J Bone Joint Surg Br 2012;94(5):584-95.

2. Klein JD, Leach KA. Pediatric pelvic osteomyelitis. Clin Pediatr (Phila) 2007;46(9):787-90.

3. Gutierrez K. Bone and joint infections in children. Pediatr Clin North Am 2005;52(3):779-94.
4. Weber-Chrysochoou C, CortiN, GoetschelP, AltermattS, et al. Pelvic osteomyelitis: a diagnostic challenge in children. J Pediatr Surg 2007;42(3):553-7.

5. Kumar J, Ramachandran M, Little D, Zenios M. Pelvic osteomyelitis in children. J Pediatr Orthop B 2010;19(1):38-41.

6. Davidson D, Letts M, Khoshhal K. Pelvic osteomyelitis in children: a comparison of decades from 1980-1989 with 1990-2001. J Pediatr Orthop 2003;23(4):514-21.

7. Hammond PJ,MacnicolMF. Osteomyelitis of the pelvis and proximal femur: diagnostic difficulties. J Pediatr Orthop B 2001;10(2):113-9.

8. Zvulunov A, Gal N, Segev Z. Acute hematogenous osteomyelitis of the pelvis in childhood: Diagnostic clues and pitfalls. Pediatr Emerg Care 2003;19(1):29-31.

9. AkhrasN, Blackwood A. Pseudomonas pelvic osteomyelitis in a healthy child. Infect Dis Rep 2011;4(1):e1.

10. PinedaC, Vargas A, RodríguezAV.Imaging of osteomyelitis: current concepts. Infect Dis Clin North Am 2006; 20(4): 789-825. 